\title{
Analytic double-soft integrated subtraction terms for two massive emitters in a back-to-back kinematics
}

\author{
Wojciech Bizon ${ }^{a, b}$ and Maximilian Delto ${ }^{a}$ \\ ${ }^{a}$ Institut für Theoretische Teilchenphysik (TTP), Karlsruhe Institute of Technology (KIT), \\ Wolfgang-Gaede Straße 1, 76128 Karlsruhe, Germany \\ ${ }^{b}$ Institut für Kernphysik (IKP), Karlsruhe Institute of Technology (KIT), \\ Hermann-von-Helmholtz-Platz 1, 76344 Eggenstein-Leopoldshafen, Germany \\ E-mail: wojciech.bizon@kit.edu, maximilian.delto@kit.edu
}

ABSTRACT: We consider the double-soft limit of QCD amplitudes with two massive quarks in a back-to-back kinematics accompanied by two soft partons. We integrate analytically the respective double-soft eikonal functions over the phase space of the two soft partons. Within the context of the nested soft-collinear subtraction scheme, our results may serve as one of the integrated subtraction terms needed for the analytic and fully-differential description of next-to-next-to-leading order (NNLO) QCD corrections to colour-singlet decay into massive partons or to heavy-quark pair production.

KEYWORDS: QCD Phenomenology

ARXIV EPRINT: 2004.01663 


\section{Contents}

1 Introduction 1

2 Preliminary remarks 3

2.1 The nested soft-collinear subtraction scheme 3

2.2 Eikonal functions for single- and double-soft emissions 5

3 Phase-space integrals $\quad 7$

$\begin{array}{lll}3.1 & \text { Single-emission integrals } & 7\end{array}$

3.2 Double-emission integrals 8

4 Results 13

5 Conclusions 16

\section{Introduction}

The focus of the physics program at the LHC has recently shifted from direct searches for new particles to precision studies of various Standard Model (SM) processes. Such studies are indispensable since, despite the discovery of the Higgs boson in $2012[1,2]$ that formally completed the SM of particle physics, there is a number of intriguing questions that cannot be answered within this theory. Given the lack of direct evidence for new particles in collider experiments, it becomes important to stress-test predictions of the SM with an unprecedented precision, which becomes possible thanks to the upcoming highluminosity phase at the LHC. As a consequence, high-precision theoretical predictions for many observables that can be studied in various SM processes at the LHC become necessary.

The perturbative description of hard scattering processes at the LHC has to overcome two main obstacles. One is the computation of multi-scale virtual amplitudes, where loop integrals over momenta of virtual particles need to be calculated. The other obstacle is the appearance of infrared singularities during phase-space integration of real corrections when one or more emissions become soft or collinear to other partons.

At next-to-leading order (NLO) in perturbative QCD, the treatment of infrared singularities was tackled long time ago with two generic methods, slicing [3] and subtraction [4]. Since nowadays, both virtual and real corrections at NLO can be calculated in a fully automated way, the applicability of these methods is limited by computing power only. The situation changes at the next-to-next-to-leading order (NNLO), where it is still being debated how to extend the well-established NLO subtraction schemes [5-8] to the next order.

Currently, theoretical predictions with NNLO QCD accuracy exist for many LHC processes. They were obtained using slicing methods that include $q_{T^{-}}[9-12]$ and $N$ jettiness [13-16] slicing; as well as subtraction schemes such as antenna subtraction [17-27], 
geometric subtraction [28], the STRIPPER framework [29-33], local analytic sector subtraction [34, 35], the CoLoRFull method [36-47] and other approaches, e.g. the projectionto-Born method [48].

Despite the large number of available subtraction and slicing schemes, it is fair to say that an optimal subtraction scheme, capable of dealing with complex processes, is yet to be designed. A set of criteria that should be considered when attempting the construction of a subtraction scheme may include physical transparency, scalability and locality as well as analyticity and efficiency. With these considerations in mind, the nested softcollinear subtraction scheme was introduced in ref. [49], building on the sector-improved residue subtraction scheme [29-31]. There it was shown that subtractions applied to gaugeinvariant scattering amplitudes, rather than to individual Feynman diagrams, can be done in a nested fashion, yielding a somewhat simpler description.

As the name suggests, subtraction schemes handle infrared singularities of real corrections by designing suitable subtraction terms for soft and collinear divergences. Properly constructed differences of real emission contributions and subtraction terms become integrable over the full phase-space in four dimensions. The subtraction terms, however, still need to be integrated in $d=4-2 \epsilon$ dimensions where soft and collinear singularities manifest themselves as $1 / \epsilon$ poles.

When the nested soft-collinear subtraction scheme is applied to massless partons, there are two genuinely double-unresolved limits that need to be addressed. These are the doublesoft limit, where two emitted partons become soft, and the triple-collinear limit, where momenta of three partons become collinear to each other. For both of these cases, integrals over the phase space of unresolved partons, subject to specific energy constraints dictated by the setup of the nested soft-collinear subtraction scheme, have been computed [50, 51]. These results facilitate an analytic and fully-differential description of colour-singlet production [52], colour-singlet decay [53] and DIS-like processes [54]. We note that these "dipole-like" building blocks should enable a fully-differential NNLO QCD description of arbitrary processes.

The structure of IR singularities changes if massive quarks are involved in a partonic process. Indeed, since there are no collinear singularities related to massive external legs, only soft singularities need to be considered. They can be subtracted using appropriate soft eikonal functions that have to be integrated over the unresolved phase space. The goal of this article is to start exploring the subtraction terms that arise in NNLO QCD calculations for processes involving massive quarks in the context of the nested soft-collinear subtraction scheme. Specifically, we compute the integrated subtraction terms which are required to describe double-soft emissions off two radiators of the same mass in a back-to-back kinematics.

The remainder of this paper is organized as follows. In section 2, we describe the nested soft-collinear subtraction scheme and the single- and the double-soft functions for massive radiators. In section 3, we integrate the single-soft and the double-soft eikonal functions over the respective unresolved phase space. We discuss results in section 4 and conclude in section 5 . 


\section{Preliminary remarks}

In this section, we specify a physical setup, describe the idea behind the nested soft-collinear subtraction scheme and establish notations by writing down the factorisation formulae for QCD amplitudes in the single-soft and the double-soft limits. We conclude the section by defining sets of single- and double-soft emission integrals that need to be computed.

Our goal is to describe infrared (IR) singularities that arise in NNLO QCD calculations for processes involving massive quarks. In this work, we focus on the IR singularities that appear in the double-real contribution to the decay of a colour-singlet particle $X$ to massive quarks, i.e. a tree-level process

$$
X \longrightarrow Q\left(p_{A}\right)+\bar{Q}\left(p_{B}\right)+f\left(k_{1}\right)+\bar{f}\left(k_{2}\right) .
$$

In eq. (2.1), $Q$ stands for a massive quark while $f$ and $\bar{f}$ denote a pair of massless partons (gluons or a quark-antiquark pair). Following ref. [49], we write the contribution of the partonic process in eq. (2.1) to the decay rate as

$$
\begin{aligned}
\left\langle\mathrm{d} \Gamma_{\mathrm{RR}}\right\rangle & =\mathcal{N} \int\left[\mathrm{d} k_{1}\right]\left[\mathrm{d} k_{2}\right] \theta\left(E_{1}-E_{2}\right) \mathrm{dLips}_{A B ; 12}|\mathcal{M}(A, B ; 1,2)|^{2} \mathcal{F}(A, B ; 1,2) \\
& =\left\langle\left[\mathrm{d} k_{1}\right]\left[\mathrm{d} k_{2}\right] F_{L M}(A, B ; 1,2)\right\rangle .
\end{aligned}
$$

In eq. (2.2), $\mathcal{N}$ includes normalization and symmetry factors, dLips ${ }_{A B ; 12}$ denotes the Lorentz invariant phase-space measure of the massive quark system, including the energymomentum conserving $\delta$-function, and $\mathcal{F}$ is the measurement function of an arbitrary infrared-safe observable. All of these quantities are then absorbed into the function $F_{L M}$ in eq. (2.2).

Note that, following the original formulation of the nested soft-collinear subtraction scheme, we have introduced an energy ordering for the radiated partons $k_{1}$ and $k_{2}$, i.e. we require $E_{1}>E_{2}$ in eq. (2.2). We define the phase-space element of a massless parton as

$$
\left[\mathrm{d} k_{i}\right]=\frac{\mathrm{d}^{d-1} k_{i}}{2 E_{i}} \theta\left(E_{\max }-E_{i}\right) .
$$

In eq. (2.3), we introduced an energy cut-off $E_{\max }$ which is arbitrary but must be large enough so that it does not change the value of the integral in eq. (2.2), see ref. [49] for details. The need for such a cut-off parameter will become clear later when the double-soft limit of eq. (2.2) is discussed. For now, we only note that $E_{\max }$ breaks Lorentz invariance but leaves rotational invariance intact.

\subsection{The nested soft-collinear subtraction scheme}

Infrared divergences of QCD amplitudes can be regulated by introducing appropriate subtraction terms for all relevant kinematic configurations. Within the nested soft-collinear subtraction scheme, such subtractions are constructed in an iterative manner, starting from the double-soft limit. 
The double-soft limit describes kinematic configurations where energies of both emissions in eq. (2.2), $E_{1}$ and $E_{2}$, vanish at a comparable rate. To describe this limit, we introduce a double-soft projection operator $\mathbb{S}$. For a generic amplitude $\mathcal{M}$ involving momenta $k_{1}$ and $k_{2}$, we consider the scaling $E_{1} \sim E_{2} \sim \lambda$ and define

$$
\mathscr{S}\left|\mathcal{M}\left(k_{1}, k_{2}\right)\right|^{2}=\lim _{\lambda \rightarrow 0} \lambda^{4}\left|\mathcal{M}\left(\lambda k_{1}, \lambda k_{2}\right)\right|^{2}
$$

We then split the double-real contribution in eq. (2.2) into double-soft regulated and unresolved parts, i.e.

$$
\begin{aligned}
\left\langle\left[\mathrm{d} k_{1}\right]\left[\mathrm{d} k_{2}\right] F_{L M}(A, B ; 1,2)\right\rangle= & \left\langle\left[\mathrm{d} k_{1}\right]\left[\mathrm{d} k_{2}\right](I-S) F_{L M}(A, B ; 1,2)\right\rangle \\
& +\left\langle\left[\mathrm{d} k_{1}\right]\left[\mathrm{d} k_{2}\right] S F_{L M}(A, B ; 1,2)\right\rangle .
\end{aligned}
$$

The first term on the right-hand side is not divergent in the double-soft limit. This term still contains singularities in the single-soft limit, where $E_{2} \rightarrow 0$, and in the collinear limit, where the two emitted partons become collinear to each other. Deriving a full subtraction would require us to remove these singularities as well. However, since integrated subtraction terms in these two limits can be obtained in a rather straightforward manner (see e.g. ref. [55]), in this paper we focus on the second term on the right-hand side of eq. (2.5) and its integration over the double-unresolved phase space. It reads

$$
\begin{aligned}
& \left\langle\left[\mathrm{d} k_{1}\right]\left[\mathrm{d} k_{2}\right] \mathscr{S} F_{L M}(A, B ; 1,2)\right\rangle \\
& \quad=\mathcal{N} \int\left[\mathrm{d} k_{1}\right]\left[\mathrm{d} k_{2}\right] \theta\left(E_{1}-E_{2}\right) \mathrm{dLips}_{A B} S \mathscr{S}|\mathcal{M}(A, B ; 1,2)|^{2} \mathcal{F}(A, B) .
\end{aligned}
$$

We note that in the double-soft limit the momenta $k_{1}$ and $k_{2}$ completely decouple from the hard matrix element, from the energy-momentum conserving $\delta$-function and from the measurement function $\mathcal{F}$. This allows us to obtain integrals over the double-unresolved phase space in a universal manner. After the decoupling from the energy-momentum conservation, integrals over $\mathrm{d} E_{1}$ and $\mathrm{d} E_{2}$ in eq. (2.6) are only limited by the cut-off parameter $E_{\max }$ introduced in eq. (2.3).

As a consequence of the factorization in the double-soft limit, the reduced matrix element describes the Born-like process

$$
X \longrightarrow Q\left(p_{A}\right)+\bar{Q}\left(p_{B}\right)
$$

and the momenta $p_{A, B}$ are back-to-back in the rest frame of the decaying particle $X$. In this kinematic situation

$$
p_{A}+p_{B}=p_{A B}=E(1,+\beta \boldsymbol{n})+E(1,-\beta \boldsymbol{n})=(2 E, \mathbf{0})
$$

and the heavy-quark momenta are on the mass shell

$$
p_{A}^{2}=p_{B}^{2}=m^{2} .
$$


We note that the quark energy $E$ is half the mass of the decaying particle, $E=M_{X} / 2$, the vector $\boldsymbol{n}$ describes the direction of flight of the heavy quark in the rest frame of the decaying colour singlet and

$$
\beta=\sqrt{1-\frac{m^{2}}{E^{2}}}
$$

The threshold limit $E=m$ implies $\beta=0$.

\subsection{Eikonal functions for single- and double-soft emissions}

Soft factorization formulas for generic QCD tree-level amplitudes involving massless radiators and up to two soft partons were studied, for example, in ref. [56]. This result was extended to cover massive radiators in ref. [30] using the observation that eikonal currents are identical for massive and massless emitters and that emitters' masses become relevant only when eikonal currents are squared.

We begin with the single-gluon emission. The limit of an amplitude that contains a gluon with a soft momentum $k$ reads

$$
\hat{S}_{k}\left|\mathcal{M}^{g}(\{p\}, k)\right|^{2}=-g_{s, b}^{2} \sum_{i, j=1}^{n} \mathcal{S}_{i j}(k)\left|\mathcal{M}^{(i j)}(\{p\})\right|^{2},
$$

where $\{p\}=\left\{p_{1}, p_{2}, \ldots, p_{n}\right\}$ and the sum runs over all $n$ hard emitters. The operator $\hat{S}_{k}$ extracts the leading asymptotic behaviour of the matrix element in the soft limit, $E_{k} \rightarrow 0$. The single-eikonal function $\mathcal{S}_{i j}(k)$ reads

$$
\mathcal{S}_{i j}(k)=\frac{\left(p_{i} \cdot p_{j}\right)}{\left(p_{i} \cdot k\right)\left(p_{j} \cdot k\right)} .
$$

The colour correlations in eq. (2.11) are encoded in the reduced matrix element ${ }^{1}$

$$
\left|\mathcal{M}^{(i j)}(\{p\})\right|^{2}=\left\langle\mathcal{M}(\{p\})\left|\boldsymbol{T}_{i} \cdot \boldsymbol{T}_{j}\right| \mathcal{M}(\{p\})\right\rangle .
$$

The double-soft function that describes emission of two gluons with momenta $k_{1}$ and $k_{2}$ reads

$$
\begin{aligned}
S^{\prime}\left|\mathcal{M}^{g g}\left(\{p\}, k_{1}, k_{2}\right)\right|^{2}=g_{s, b}^{4}\{ & \frac{1}{2} \sum_{i, j, k, l=1}^{n} \mathcal{S}_{i j}\left(k_{1}\right) \mathcal{S}_{k l}\left(k_{2}\right)\left|\mathcal{M}^{\{(i j),(k l)\}}(\{p\})\right|^{2} \\
& \left.-C_{A} \sum_{i, j=1}^{n} \mathcal{S}_{i j}\left(k_{1}, k_{2}\right)\left|\mathcal{M}^{(i j)}(\{p\})\right|^{2}\right\},
\end{aligned}
$$

where the additional colour correlated matrix element is defined as

$$
\left|\mathcal{M}^{\{(i j),(k l)\}}(\{p\})\right|^{2}=\left\langle\mathcal{M}\left(p_{1}, \ldots, p_{n}\right)\left|\left\{\boldsymbol{T}_{i} \cdot \boldsymbol{T}_{j}, \boldsymbol{T}_{k} \cdot \boldsymbol{T}_{l}\right\}\right| \mathcal{M}\left(p_{1}, \ldots, p_{n}\right)\right\rangle,
$$

and the notation $\{\cdot, \cdot\}$ stands for an anticommutator in colour space. The first term on the right-hand side of eq. (2.14) is the abelian contribution. It is simply a product of singleeikonal factors defined in eq. (2.12). Due to its factorized form, it is particularly easy to

\footnotetext{
${ }^{1}$ To describe colour degrees of freedom we use colour-space notation from ref. [7].
} 
integrate this term over the soft-gluons phase space. The second, non-abelian contribution is proportional to the colour factor $C_{A}$. It is given by the function $\mathcal{S}_{i j}\left(k_{1}, k_{2}\right)$ which reads

$$
\mathcal{S}_{i j}\left(k_{1}, k_{2}\right)=\mathcal{S}_{i j}^{0}\left(k_{1}, k_{2}\right)+\left[m_{i}^{2} \mathcal{S}_{i j}^{m}\left(k_{1}, k_{2}\right)+m_{j}^{2} \mathcal{S}_{j i}^{m}\left(k_{1}, k_{2}\right)\right]
$$

where we note that the term in square brackets explicitly depends on the squared masses of the emitters $m_{i}^{2}$ and $m_{j}^{2}$. Both $\mathcal{S}_{i j}^{0}\left(k_{1}, k_{2}\right)$ and $\mathcal{S}_{i j}^{m}\left(k_{1}, k_{2}\right)$ implicitly depend on the masses. The first term in eq. $(2.16), \mathcal{S}_{i j}^{0}\left(k_{1}, k_{2}\right)$, also appears in the factorization formula for massless emitters [56]; it reads

$$
\begin{aligned}
\mathcal{S}_{i j}^{0}\left(k_{1}, k_{2}\right)= & \frac{(1-\epsilon)}{\left(k_{1} \cdot k_{2}\right)^{2}} \frac{\left[\left(p_{i} \cdot k_{1}\right)\left(p_{j} \cdot k_{2}\right)+i \leftrightarrow j\right]}{\left(p_{i} \cdot k_{12}\right)\left(p_{j} \cdot k_{12}\right)} \\
& -\frac{\left(p_{i} \cdot p_{j}\right)^{2}}{2\left(p_{i} \cdot k_{1}\right)\left(p_{j} \cdot k_{2}\right)\left(p_{i} \cdot k_{2}\right)\left(p_{j} \cdot k_{1}\right)}\left[2-\frac{\left[\left(p_{i} \cdot k_{1}\right)\left(p_{j} \cdot k_{2}\right)+i \leftrightarrow j\right]}{\left(p_{i} \cdot k_{12}\right)\left(p_{j} \cdot k_{12}\right)}\right] \\
& +\frac{\left(p_{i} \cdot p_{j}\right)}{2\left(k_{1} \cdot k_{2}\right)}\left[\frac{2}{\left(p_{i} \cdot k_{1}\right)\left(p_{j} \cdot k_{2}\right)}+\frac{2}{\left(p_{j} \cdot k_{1}\right)\left(p_{i} \cdot k_{2}\right)}-\frac{1}{\left(p_{i} \cdot k_{12}\right)\left(p_{j} \cdot k_{12}\right)}\right. \\
& \left.\times\left(4+\frac{\left[\left(p_{i} \cdot k_{1}\right)\left(p_{j} \cdot k_{2}\right)+i \leftrightarrow j\right]^{2}}{\left(p_{i} \cdot k_{1}\right)\left(p_{j} \cdot k_{2}\right)\left(p_{i} \cdot k_{2}\right)\left(p_{j} \cdot k_{1}\right)}\right)\right] .
\end{aligned}
$$

The other two contributions in eq. (2.16) are only relevant for massive hard emitters. The function $\mathcal{S}_{i j}^{m}\left(k_{1}, k_{2}\right)$ is given by [30]

$$
\begin{aligned}
\mathcal{S}_{i j}^{m}\left(k_{1}, k_{2}\right)= & -\frac{1}{4\left(k_{1} \cdot k_{2}\right)\left(p_{i} \cdot k_{1}\right)\left(p_{i} \cdot k_{2}\right)}+\frac{\left(p_{i} \cdot p_{j}\right)\left(p_{j} \cdot k_{12}\right)}{2\left(p_{i} \cdot k_{1}\right)\left(p_{j} \cdot k_{2}\right)\left(p_{i} \cdot k_{2}\right)\left(p_{j} \cdot k_{1}\right)\left(p_{i} \cdot k_{12}\right)} \\
& -\frac{1}{2\left(k_{1} \cdot k_{2}\right)\left(p_{i} \cdot k_{12}\right)\left(p_{j} \cdot k_{12}\right)}\left(\frac{\left(p_{j} \cdot k_{1}\right)^{2}}{\left(p_{i} \cdot k_{1}\right)\left(p_{j} \cdot k_{2}\right)}+\frac{\left(p_{j} \cdot k_{2}\right)^{2}}{\left(p_{i} \cdot k_{2}\right)\left(p_{j} \cdot k_{1}\right)}\right) .
\end{aligned}
$$

Note that we use an abbreviation $k_{12}=k_{1}+k_{2}$ in eqs. (2.17) and (2.18).

When a soft quark-antiquark pair is emitted, the soft limit of the matrix element is described by

$$
S_{S}\left|\mathcal{M}^{q \bar{q}}\left(\{p\}, k_{1}, k_{2}\right)\right|^{2}=g_{s, b}^{4} T_{F} \sum_{i, j=1}^{n} \mathcal{I}_{i j}\left(k_{1}, k_{2}\right)\left|\mathcal{M}^{(i j)}(\{p\})\right|^{2},
$$

where $T_{F}=1 / 2$. The soft function $\mathcal{I}_{i j}\left(k_{1}, k_{2}\right)$ is given by

$$
\mathcal{I}_{i j}\left(k_{1}, k_{2}\right)=\frac{\left[\left(p_{i} \cdot k_{1}\right)\left(p_{j} \cdot k_{2}\right)+i \leftrightarrow j\right]-\left(p_{i} \cdot p_{j}\right)\left(k_{1} \cdot k_{2}\right)}{\left(k_{1} \cdot k_{2}\right)^{2}\left(p_{i} \cdot k_{12}\right)\left(p_{j} \cdot k_{12}\right)} .
$$

As we already mentioned, in the soft limit the dependence on the soft gluon momenta drops out from the matrix element as well as from the momentum conserving $\delta$-function. For this reason, the eikonal factors in eqs. (2.14) and (2.19) can be integrated over the soft-gluons phase space, irrespective of matrix elements that describe the underlying hard process. 
In the following, we explain how to do that in the case of two equal mass emitters whose momenta $p_{A}$ and $p_{B}$ are back-to-back. To simplify notations, we introduce the single-emission phase-space integral

$$
\mathcal{G}_{i j}=\int[\mathrm{d} k] \mathcal{S}_{i j}(k)
$$

where $i j \in\{A A, A B, B A, B B\}$ and the phase-space measure and eikonal functions are defined in eqs. (2.3) and (2.12), respectively. For the double-emission phase-space integrals, we distinguish between emissions of gluons and quarks and define

$$
\begin{aligned}
\mathcal{G} \mathcal{G}_{i j} & =\int_{E_{2}<E_{1}}\left[\mathrm{~d} k_{1}\right]\left[\mathrm{d} k_{2}\right] \mathcal{S}_{i j}\left(k_{1}, k_{2}\right), \\
\mathcal{Q} \overline{\mathcal{Q}}_{i j} & =\int_{E_{2}<E_{1}}\left[\mathrm{~d} k_{1}\right]\left[\mathrm{d} k_{2}\right] \mathcal{I}_{i j}\left(k_{1}, k_{2}\right),
\end{aligned}
$$

where, again, ij $\in\{A A, A B, B A, B B\}$. We note that in case of back-to-back kinematics, integrated subtraction terms $B B$ and $A A$, as well as $B A$ and $A B$, are equal to each other. Therefore, in what remains, we will only consider cases $i j=A A$ and $i j=A B$.

The integrals in eqs. (2.21) and (2.22) fully describe the integrated soft subtraction terms in the decay process of eq. (2.1) and are an important ingredient for more complex processes, such as heavy-quark pair production. The computation of phase-space integrals in eq. (2.22) is the main goal of this paper. We describe the details of the computation in the following section. We note that a similar calculation was performed in ref. [57], however, the unresolved phase space in that paper was subject to a slightly different constraint.

\section{Phase-space integrals}

In this section we present details of the calculation of the integrals defined in eqs. (2.21) and (2.22). We start with the single-soft emission to clarify notation and then proceed to the double-emission case. The results of the latter calculation are discussed in section 4 .

\subsection{Single-emission integrals}

We start with a brief discussion of single-emission integrals. The first integral reads

$$
\begin{aligned}
\mathcal{G}_{A B} & =\int[\mathrm{d} k] \frac{\left(p_{A} \cdot p_{B}\right)}{\left(p_{A} \cdot k\right)\left(p_{B} \cdot k\right)} \\
& =\frac{\left(1+\beta^{2}\right)}{2} \int_{0}^{E_{\max }} \frac{\mathrm{d} E}{E^{1+2 \epsilon}} \int \frac{\mathrm{d} \Omega_{k}^{(d-1)}}{\left(1-\beta \boldsymbol{n} \cdot \boldsymbol{n}_{k}\right)\left(1+\beta \boldsymbol{n} \cdot \boldsymbol{n}_{k}\right)},
\end{aligned}
$$

where we have parametrised the gluon four momentum as $k=E\left(1, \boldsymbol{n}_{k}\right)$. Further, we choose the reference frame in such a way that the $z$-axis points in the $\boldsymbol{n}$ direction. This yields $\left(1 \pm \beta \boldsymbol{n} \cdot \boldsymbol{n}_{k}\right)=(1 \pm \beta \cos \theta)$ and, after introducing $\eta=(1-\cos \theta) / 2$, we obtain

$$
\mathcal{G}_{A B}=-\frac{\left(1+\beta^{2}\right) E_{\max }^{-2 \epsilon}}{4 \epsilon} \times \Omega^{(d-2)} \int_{0}^{1} \mathrm{~d} \eta\left(\frac{[4 \eta(1-\eta)]^{-\epsilon}}{[1-\beta(1-2 \eta)]}+\frac{[4 \eta(1-\eta)]^{-\epsilon}}{[1+\beta(1-2 \eta)]}\right)
$$


where $\Omega^{(n)}=2 \pi^{n / 2} / \Gamma(n / 2)$ denotes the surface of a unit sphere embedded in $n$ dimensions. The integral in eq. (3.2) can be written as a hypergeometric function of the type ${ }_{2} F_{1}[\{a, b\},\{2 b\} ; z]$, which further simplifies to [58]

$$
{ }_{2} F_{1}[\{a, b\},\{2 b\} ; z]=(1-z / 2)^{-a}{ }_{2} F_{1}\left[\{a / 2+1 / 2, a / 2\},\{b+1 / 2\} ; z^{2} /(2-z)^{2}\right] .
$$

We find

$$
\mathcal{G}_{A B}=-\frac{\left(1+\beta^{2}\right) E_{\max }^{-2 \epsilon}}{4 \epsilon} \times \Omega^{(d-1)} \times{ }_{2} F_{1}\left[\{1,1 / 2\},\{3 / 2-\epsilon\} ; \beta^{2}\right] .
$$

The second integral, for a self-correlated emission, reads

$$
\begin{aligned}
\mathcal{G}_{A A} & =\int[\mathrm{d} k] \frac{m^{2}}{\left(p_{A} \cdot k\right)^{2}} \\
& =-\frac{E_{\max }^{-2 \epsilon}}{4 \epsilon} \times \Omega^{(d-1)} \times\left(1-2 \epsilon+2 \epsilon_{2} F_{1}\left[\{1,1 / 2\},\{3 / 2-\epsilon\} ; \beta^{2}\right]\right) .
\end{aligned}
$$

Note that the hypergeometric function which appears in eqs. (3.4) and (3.5) features an expansion in powers of $\epsilon$ in terms of classical polylogarithms with arguments that involve square roots of $\beta$. In order to simplify the expansion, we again rewrite the hypergeometric function [58] and find

$$
\begin{aligned}
& { }_{2} F_{1}\left[\{1,1 / 2\},\{3 / 2-\epsilon\} ; \beta^{2}\right]=\frac{1-2 \epsilon}{2 \epsilon \beta}\left(\frac{2 \beta}{1+\beta}\right)^{2 \epsilon} \times \\
& \quad \times\left\{\left(\frac{1-\beta}{1+\beta}\right)^{-\epsilon} \frac{\Gamma(1-2 \epsilon) \Gamma(1+\epsilon)}{\Gamma(1-\epsilon)}-{ }_{2} F_{1}\left[\{\epsilon, 2 \epsilon\},\{1+\epsilon\} ; \frac{1-\beta}{1+\beta}\right]\right\} .
\end{aligned}
$$

Using HypExp [59], the hypergeometric function in eq. (3.6) can be expanded as

$$
\begin{aligned}
{ }_{2} F_{1}\left[\{\epsilon, 2 \epsilon\},\{1+\epsilon\} ; \frac{1-\beta}{1+\beta}\right] \\
=1+2 \epsilon^{2} \operatorname{Li}_{2}\left(\frac{1-\beta}{1+\beta}\right)+\epsilon^{3}\left[4 \zeta_{3}+\frac{2 \pi^{2}}{3} \ln \left(\frac{2 \beta}{1+\beta}\right)-2 \ln \left(\frac{1-\beta}{1+\beta}\right) \ln ^{2}\left(\frac{2 \beta}{1+\beta}\right)\right. \\
\left.\quad-4 \ln \left(\frac{2 \beta}{1+\beta}\right) \operatorname{Li}_{2}\left(\frac{1-\beta}{1+\beta}\right)-2 \operatorname{Li}_{3}\left(\frac{1-\beta}{1+\beta}\right)-4 \operatorname{Li}_{3}\left(\frac{2 \beta}{1+\beta}\right)\right]+\mathcal{O}\left(\epsilon^{4}\right) .
\end{aligned}
$$

The results shown in eqs. (3.4) and (3.5) were derived earlier in the literature [60, 61].

\subsection{Double-emission integrals}

We now turn to the calculation of the double-soft subtraction terms. We need to compute the four functions $\mathcal{G} \mathcal{G}_{A A}, \mathcal{G G} \mathcal{G}_{A B}, \mathcal{Q} \overline{\mathcal{Q}}_{A A}$ and $\mathcal{Q} \overline{\mathcal{Q}}_{A B}$ in eq. (2.22). To this end we employ reverse unitarity [62] that has been previously used for the computation of other integrated subtraction terms $[50,51]$.

Computational setup. The integration measure for the two energy-ordered emissions in eq. (2.22) reads

$$
\left.\left[\mathrm{d} k_{1}\right]\left[\mathrm{d} k_{2}\right]\right|_{E_{2}<E_{1}}=\frac{\mathrm{d}^{d-1} k_{1}}{2 E_{1}} \frac{\mathrm{d}^{d-1} k_{2}}{2 E_{2}} \theta\left(E_{\max }-E_{1}\right) \theta\left(E_{1}-E_{2}\right) .
$$


Note that all integrands, $\mathcal{S}_{i j}\left(k_{1}, k_{2}\right)$ and $\mathcal{I}_{i j}\left(k_{1}, k_{2}\right)$, are homogeneous under uniform rescaling of $E_{1}$ and $E_{2}$. For this reason we parametrise the energies as

$$
E_{1}=E_{\max } \cdot x, \quad E_{2}=E_{\max } \cdot x \cdot z,
$$

and integrate over $x$ to obtain

$$
\begin{aligned}
\mathcal{G} \mathcal{G}_{i j} & =-\frac{E_{\max }^{-4 \epsilon}}{16 \epsilon} \int_{0}^{1} \mathrm{~d} z z^{1-2 \epsilon} \int \mathrm{d} \Omega_{12}^{(d-1)} \mathcal{S}_{i j}\left(n_{1}, z \cdot n_{2}\right), \\
\mathcal{Q} \overline{\mathcal{Q}}_{i j} & =-\frac{E_{\max }^{-4 \epsilon}}{16 \epsilon} \int_{0}^{1} \mathrm{~d} z z^{1-2 \epsilon} \int \mathrm{d} \Omega_{12}^{(d-1)} \mathcal{I}_{i j}\left(n_{1}, z \cdot n_{2}\right),
\end{aligned}
$$

where $n_{i}=\left(1, \boldsymbol{n}_{i}\right)$, and the angular integration measure reads $\mathrm{d} \Omega_{12}^{(d-1)}=\mathrm{d} \Omega_{1}^{(d-1)} \mathrm{d} \Omega_{2}^{(d-1)}$.

It remains to carry out angular and $z$ integrations in eqs. (3.10) and (3.11). However, the gluon emission case exhibits an additional singularity in the strongly-ordered limit, where the gluon with momentum $k_{2}$ is much softer than the gluon with momentum $k_{1}$. Such behaviour results in a logarithmic divergence in the $z$ integration at $z=0$, which prevents us from a naive Taylor expansion of the integrand in $\epsilon$. The problem can be ameliorated by using endpoint subtraction at $z=0$. To accomplish this, we extract the divergent part using the following formula

$$
\mathcal{S}_{i j}^{\text {s.o. }}\left(n_{1}, n_{2}\right)=z^{-2} \lim _{z \rightarrow 0}\left[z^{2} \mathcal{S}_{i j}\left(n_{1}, z \cdot n_{2}\right)\right]
$$

We note that it is beneficial to perform such a subtraction at the level of the full integrand since the resulting expression fully accounts for gauge properties of QCD amplitudes and, in variance to individual integrals, does not exhibit unphysical singularities.

Note that an emission of a soft quark-antiquark pair does not exhibit the $z \rightarrow 0$ singularity and, for this reason, does not require additional subtraction.

To perform angular integrals in eqs. (3.10) and (3.11) we proceed as follows. In the spirit of reverse unitarity [62], we rewrite $\delta$-functions through cut propagators. To this end, we first rewrite the angular integration measures for both emissions as

$$
\mathrm{d} \Omega_{i}^{(d-1)}=4 \mathrm{~d}^{d} k_{i} \delta^{+}\left(k_{i}^{2}\right) \delta\left(\left(k_{i} \cdot p_{A B}\right)-\xi_{i} p_{A B}^{2} / 2\right)\left(p_{A B}^{2} / 4\right)^{\epsilon} \xi_{i}^{-1+2 \epsilon}, \quad i=1,2,
$$

with $\xi_{1}=1$ and $\xi_{2}=z$. By applying Cutkosky rules [63] backwards, we define cut loop integrals

$$
\begin{aligned}
& \mathcal{E}_{i j}^{\mathcal{G G}}(z, \beta, \epsilon)=\int \frac{\mathrm{d}^{d} k_{1} \mathrm{~d}^{d} k_{2} \mathcal{S}_{i j}\left(k_{1}, k_{2}\right)}{\left[k_{1}^{2}\right]_{c}\left[k_{2}^{2}\right]_{c}\left[k_{1} \cdot p_{A B}-2 E^{2}\right]_{c}\left[k_{2} \cdot p_{A B}-2 E^{2} z\right]_{c}}, \\
& \mathcal{E}_{i j}^{\mathcal{G G}, \text {,s.o. }}(z, \beta, \epsilon)=\int \frac{\mathrm{d}^{d} k_{1} \mathrm{~d}^{d} k_{2} \mathcal{S}_{i j}^{\text {s.o. }}\left(k_{1}, k_{2}\right)}{\left[k_{1}^{2}\right]_{c}\left[k_{2}^{2}\right]_{c}\left[k_{1} \cdot p_{A B}-2 E^{2}\right]_{c}\left[k_{2} \cdot p_{A B}-2 E^{2} z\right]_{c}}, \\
& \mathcal{E}_{i j}^{\mathcal{Q}} \overline{\mathcal{Q}}(z, \beta, \epsilon)=\int \frac{\mathrm{d}^{d} k_{1} \mathrm{~d}^{d} k_{2} \mathcal{I}_{i j}\left(k_{1}, k_{2}\right)}{\left[k_{1}^{2}\right]_{c}\left[k_{2}^{2}\right]_{c}\left[k_{1} \cdot p_{A B}-2 E^{2}\right]_{c}\left[k_{2} \cdot p_{A B}-2 E^{2} z\right]_{c}} .
\end{aligned}
$$


We note that the variable $z$ appears only in one of the cut propagators and plays the role of an internal mass. We use the definitions of eq. (3.14) in eqs. (3.10) and (3.11) and write

$$
\begin{aligned}
& \mathcal{G} \mathcal{G}_{i j}=-\frac{1}{\epsilon}\left(\frac{E_{\text {max }}}{E}\right)^{-4 \epsilon} {\left[\int_{0}^{1} \mathrm{~d} z\left(\mathcal{E}_{i j}^{\mathcal{G G}}(z, \beta, \epsilon)-\mathcal{E}_{i j}^{\mathcal{G G}, \text { s.o. }}(z, \beta, \epsilon)\right)\right.} \\
&\left.+\int_{0}^{1} \mathrm{~d} z \mathcal{E}_{i j}^{\mathcal{G} \mathcal{G}, \text { s.o. }}(z, \beta, \epsilon)\right], \\
& \mathcal{Q} \overline{\mathcal{Q}}_{i j}=-\frac{1}{\epsilon}\left(\frac{E_{\text {max }}}{E}\right)^{-4 \epsilon} \int_{0}^{1} \mathrm{~d} z \mathcal{E}_{i j}^{\mathcal{Q} \overline{\mathcal{Q}}_{(}(z, \beta, \epsilon) .}
\end{aligned}
$$

After mapping angular integrals onto ordinary loop integrals with cut propagators, we employ standard techniques of loop calculations to compute the integrals that appear in eqs. (3.15) and (3.16).

IBP reduction. We apply integration-by-parts (IBP) techniques [64] to the integrands of eqs. (3.15) and (3.16) to express them in terms of a few master integrals. The integrands consist of two-loop cut integrals

$$
T^{a_{1}, a_{2}, a_{3}}\left(\alpha_{1}, \alpha_{2}, \alpha_{3}\right)=\left(E^{2}\right)^{-d+4+\sum_{i=1}^{3} \alpha_{i}} \int \frac{\mathrm{d}^{d} k_{1} \mathrm{~d}^{d} k_{2}}{D_{\text {cut }} D_{a_{1}}^{\alpha_{1}} D_{a_{2}}^{\alpha_{2}} D_{a_{3}}^{\alpha_{3}}} \equiv\left\langle\prod_{i=1}^{3} \frac{1}{D_{a_{i}}^{\alpha_{i}}}\right\rangle
$$

where the propagators to be cut are given by

$$
D_{\text {cut }}=\left[k_{1}^{2}\right]_{c}\left[k_{2}^{2}\right]_{c}\left[k_{1} \cdot p_{A B}-2 E^{2}\right]_{c}\left[k_{2} \cdot p_{A B}-2 E^{2} z\right]_{c},
$$

and the three ordinary propagators $D_{a_{i}}$ per topology $T^{a_{1}, a_{2}, a_{3}}$ are drawn from a set

$$
D_{1, \ldots, 7}=\left\{\left(p_{A} \cdot k_{1}\right),\left(p_{B} \cdot k_{1}\right),\left(p_{A} \cdot k_{2}\right),\left(p_{B} \cdot k_{2}\right),\left(k_{1} \cdot k_{2}\right),\left(p_{A} \cdot k_{12}\right),\left(p_{B} \cdot k_{12}\right)\right\} .
$$

The variables $\alpha_{i}$ in eq. (3.17) refer to powers of propagators in integrals in a certain topology $T^{a_{1}, a_{2}, a_{3}}$. The prefactor in eq. (3.17) was chosen to render integrals dimensionless. To express all integrals in eq. (3.14) through these topologies, we use the following list of linear relations between propagators

$$
\begin{array}{ll}
D_{1}+D_{3}=D_{6}, & D_{2}+D_{4}=D_{7}, \\
D_{1}+D_{2}=2 E^{2}, & D_{3}+D_{4}=2 E^{2} z,
\end{array}
$$

where the last two equations follow from the cut constraints.

We use Reduze2 [65] to express integrals shown in eq. (3.14) through master integrals. We write

$$
\mathcal{E}_{i j}^{\mathcal{X} \mathcal{X}}(z, \beta, \epsilon)=\boldsymbol{R}_{i j}^{\mathcal{X} \mathcal{X}}(z, \beta, \epsilon) \cdot \boldsymbol{I}(z, \beta, \epsilon),
$$

where $\boldsymbol{R}_{i j}^{\mathcal{X}}(z, \beta, \epsilon)$ are vectors of reduction coefficients and $\boldsymbol{I}(z, \beta, \epsilon)$ stands for a vector constructed out of thirteen master integrals grouped into five topologies. The first integral is the phase-space volume

$$
I_{1}=\langle 1\rangle=z^{1-2 \epsilon} \frac{\left(\Omega^{(d-1)}\right)^{2}}{16}
$$


and the remaining twelve integrals are given by

$$
\begin{aligned}
I_{2, \ldots, 4} & =\left\{\left\langle\frac{1}{D_{3}}\right\rangle,\left\langle\frac{1}{D_{2} D_{3}}\right\rangle,\left\langle\frac{1}{D_{2} D_{3} D_{5}}\right\rangle\right\} \subset T^{2,3,5}, \\
I_{5, \ldots, 9} & =\left\{\left\langle\frac{D_{2}}{D_{6}}\right\rangle,\left\langle\frac{D_{5}}{D_{6}}\right\rangle,\left\langle\frac{1}{D_{6}}\right\rangle,\left\langle\frac{1}{D_{2} D_{6}}\right\rangle,\left\langle\frac{1}{D_{2} D_{5} D_{6}}\right\rangle\right\} \subset T^{2,5,6}, \\
I_{10} & =\left\{\left\langle\frac{1}{D_{2} D_{7}}\right\rangle\right\} \subset T^{2,5,7}, \\
I_{11,12} & =\left\{\left\langle\frac{1}{D_{4} D_{6}}\right\rangle,\left\langle\frac{1}{D_{4} D_{5} D_{6}}\right\rangle\right\} \subset T^{4,5,6}, \\
I_{13} & =\left\{\left\langle\frac{1}{D_{4} D_{7}}\right\rangle\right\} \subset T^{4,5,7} .
\end{aligned}
$$

We note that the gluon emission contribution $\mathcal{E}_{i j}^{\mathcal{G G}}(z, \beta, \epsilon)$ requires the full set of master integrals. On the other hand, the strongly ordered contribution $\mathcal{E}_{i j}^{\mathcal{G G}, \text { s.o. }}(z, \beta, \epsilon)$ requires master integrals $I_{1, \ldots, 4}$ and the quark-emission contribution $\mathcal{E}_{i j}^{\mathcal{Q}} \overline{\mathcal{Q}}(z, \beta, \epsilon)$ only depends on integrals $I_{1,5, \ldots, 7}$.

Differential equations. Having obtained a set of master integrals we employ the method of differential equations [66-68] to compute them. To this end, we derive a closed system of first order partial differential equations for the master integrals $\boldsymbol{I}$ as functions of variables $\beta$ and $z$. We then cast the differential equations into the $\epsilon$-homogeneous form [69] by changing the basis of master integrals

$$
\boldsymbol{I}=\hat{T}_{\text {can }} \boldsymbol{J}
$$

Here $\hat{T}_{\text {can }}$ is the transformation that brings master integrals into their so-called canonical basis $\boldsymbol{J}$. In general, finding a canonical basis or, equivalently, constructing a transformation $\hat{T}_{\text {can }}$ is a complicated task. In our case, we accomplish this by using the algorithmic approach suitable for multi-scale problems proposed in ref. [70] and implemented in the CANONICA package [71] for Mathematica. This transformation can also found using the approach of ref. [72] implemented in a private Mathematica tool Libra. ${ }^{2}$ In this case, a sequential application of the algorithm of ref. [72] is required.

In the canonical basis $\boldsymbol{J}$, differential equations take the $\epsilon$-homogeneous form

$$
\partial_{x} \boldsymbol{J}=\epsilon \hat{M}_{x} \boldsymbol{J}
$$

with $x \in\{z, \beta\}$. The matrices $\hat{M}_{z}$ and $\hat{M}_{\beta}$ feature simple poles and can be written as

$$
\hat{M}_{x}=\sum_{x_{i} \in \mathcal{A}_{x}} \frac{\hat{m}_{x_{i}}}{x-x_{i}} .
$$

\footnotetext{
${ }^{2}$ We wish to thank Roman Lee for giving us access to the Libra package.
} 
In eq. (3.26), the residue matrices $\hat{m}_{x_{i}}$ are composed of rational numbers and the poles $x_{i}$ are drawn from the two alphabets

$$
\begin{aligned}
& \mathcal{A}_{z}=\left\{0,-1, \frac{-2}{1 \pm \beta},-\frac{(1 \pm \beta)}{2},-\frac{1-\beta}{1+\beta},-\frac{1+\beta}{1-\beta}\right\}, \\
& \mathcal{A}_{\beta}=\left\{0, \pm 1, \pm(1+2 z), \pm \frac{1+z}{1-z}, \pm \frac{2+z}{z}\right\} .
\end{aligned}
$$

Thanks to the $\epsilon$-homogeneous form of the differential equations in eq. (3.25), the $\epsilon$ expansion of the functions $\boldsymbol{J}(z, \beta)$ can be obtained by recursive integration of the righthand side. Since matrices $\hat{M}_{x}$ contain only simple poles, the result can be expressed in terms of linear combinations of Goncharov Polylogarithms (GPLs) [73] that depend on $z$ and $\beta$ and constants of integration. Note that, since we are interested in a final integration over the variable $z$ in eqs. (3.15) and (3.16), it is beneficial to write master integrals in such a way that $z$ appears only as an argument of the GPLs. For this reason, at each order in $\epsilon$, we first integrate the system of differential equations with respect to $z$. A constant of integration in this case is an unspecified function of $\beta$. To determine this function, we substitute the solution into the differential equations in $\beta$, and explicitly check that the resulting differential equations are $z$ independent. After integration over $\beta$, all master integrals are expressed in terms of GPLs, $\mathrm{G}\left(\left\{\vec{z}_{0}\right\} ; z\right)$ and $\mathrm{G}\left(\left\{\vec{\beta}_{0}\right\} ; \beta\right)$, where the elements of $\vec{z}_{0}$ are drawn from the alphabet $\mathcal{A}_{z}$, cf. eq. (3.27), and elements in $\vec{\beta}_{0}$ belong to the $z$-independent part of the alphabet $\mathcal{A}_{\beta}$ in eq. (3.28), i.e. $\tilde{\mathcal{A}}_{\beta}=\{0,-1,+1\}$.

This concludes the computation of master integrals up to constants of integration. These constants are determined by calculating suitable boundary conditions as we discuss in the next section.

Boundary conditions. We find it suitable to determine constants of integration by computing master integrals in the threshold limit $\beta \rightarrow 0$. This limit is particularly convenient, since many of the integrals simplify. This happens because in that limit the dependencies of all scalar products on quark momenta disappear. For example

$$
p_{A} \cdot\left(k_{1}+k_{2}\right)=E^{2}\left[(1+z)-\beta \boldsymbol{n}\left(\boldsymbol{n}_{1}+z \boldsymbol{n}_{2}\right)\right] \stackrel{\beta \rightarrow 0}{\longrightarrow} E^{2}(1+z) .
$$

By inspecting master integrals in eq. (3.23), we observe that

$$
\lim _{\beta \rightarrow 0} \boldsymbol{I}(z, \beta, \epsilon)=\boldsymbol{F}(z, \epsilon)+\mathcal{O}(\beta) .
$$

Moreover, we find that all entries, except for the first diagonal element of the canonical transformation matrix $\hat{T}_{\text {can }}^{-1}$ are suppressed as $\mathcal{O}(\beta)$ and therefore vanish in the threshold limit. The transformation matrix in the threshold limit reads

$$
\lim _{\beta \rightarrow 0} \hat{T}_{\text {can }}^{-1}=\left(\begin{array}{ccc}
1 / z & 0 & \cdots \\
0 & 0 & \cdots \\
\vdots & \vdots & \ddots
\end{array}\right) .
$$

This means that to fix all integration constants we only need the phase-space master integral $I_{1}$, which is straightforward to compute, cf. eq. (3.22). 
After fixing all the integration constants using boundary conditions, we transform master integrals $\boldsymbol{J}$ into the original basis $\boldsymbol{I}$. We check the resulting expressions numerically for several values of $\beta$ and $z$.

Integration over $\boldsymbol{z}$. Having computed the required master integrals, we obtain the integrands in eqs. (3.15) and (3.16) and perform the integration over $z$. The masters integrals $\boldsymbol{I}$ of eqs. (3.22) and (3.23) allow us to express the functions $\mathcal{E}_{i j}^{\mathcal{X}}(z, \beta, \epsilon)$ in terms of rational functions of $z, \beta$ and GPLs of $z$ and $\beta$ with $z$-independent letters. Such a representation enables the final $z$ integration in eqs. (3.15) and (3.16) in a straightforward manner. We note that after obtaining the primitive, the $z \rightarrow 0$ limit features spurious $1 / z^{n}$ poles and needs to be taken with care. We use PolyLogTools [74] to expand all GPLs around $z=0$ up to the order required to cancel these $1 / z^{n}$ poles and facilitate $z$-integration over the interval $0<z<1$. We report results for the functions $\mathcal{G G}_{A A}, \mathcal{G G}_{A B}$, $\mathcal{Q} \overline{\mathcal{Q}}_{A A}$ and $\mathcal{Q} \overline{\mathcal{Q}}_{A B}$ in the next section.

\section{Results}

In this section, we present some results for the integrated double-soft subtraction terms, cf. eq. (2.22). We write

$$
\begin{aligned}
\mathcal{G} \mathcal{G}_{i j} & =\frac{E_{\max }^{-4 \epsilon}}{16}\left(\Omega^{(d-1)}\right)^{2} \times f_{i j}^{g g}(\beta, \epsilon), \\
\mathcal{Q} \overline{\mathcal{Q}}_{i j} & =\frac{E_{\max }^{-4 \epsilon}}{16}\left(\Omega^{(d-1)}\right)^{2} \times f_{i j}^{q \bar{q}}(\beta, \epsilon),
\end{aligned}
$$

with $\Omega^{(n)}$ defined after eq. (3.2). Four results for functions $f_{i j}^{g g, q \bar{q}}(\beta, \epsilon)$ can be found in the supplementary material provided with this submission. They are expressed through GPLs of $\beta$ up to weight four, with integer letters drawn from the alphabet

$$
\mathcal{A}=\{0, \pm 1, \pm 3\}
$$

We use a private implementation of the super-shuffle identities described in ref. [75] to translate the expressions obtained from the integration over $z$, cf. section 3.2, into such a fibration basis. We note that all GPLs appearing in eq. (4.1) are manifestly real in the physical region $\beta \in[0,1]$. For a numerical evaluation of GPLs one can resort to publicly available programs $[76,77]$. The functions $f_{i j}^{g g, q \bar{q}}(\beta, \epsilon)$ were checked numerically using an adaptation of the numerical routine from ref. [55].

While functions $f_{A A}^{g g}$ and $f_{A B}^{g g}$, that describe the emission of two soft gluons, feature $1 / \epsilon^{3}$ poles, the functions $f_{A A}^{q \bar{q}}$ and $f_{A B}^{q \bar{q}}$, related to quark pair emissions, start only at $1 / \epsilon^{2}$. This happens because the latter case does not exhibit a strongly ordered soft divergence, cf. eq. (3.16). The expressions for $1 / \epsilon$ poles of the functions $f_{i j}^{g g, q \bar{q}}(\beta, \epsilon)$ consist only of harmonic polylogarithms (HPLs) [78] of $\beta$ up to weight three. We rewrite them in terms 
of independent classical polylogarithms [79] and find

$$
\begin{aligned}
& f_{A A}^{g g}(\beta, \epsilon)=-\frac{1}{8 \epsilon^{3}}+\frac{1}{\epsilon^{2}} \frac{1}{4 \beta}\left\{\ln \left(x_{\beta}\right)+\beta\right\}+\frac{1}{\epsilon} \frac{1}{4 \beta}\left\{2 \beta-3 \ln \left(x_{\beta}\right)-8 \beta \ln (2)\right. \\
& -2\left[\operatorname{Li}_{2}\left(y_{\beta}^{-}\right)+\operatorname{Li}_{2}(\beta)-\mathrm{Li}_{2}(-\beta)\right]+y_{\beta}^{-} \ln ^{2}\left(x_{\beta}\right) \\
& \left.-\ln ^{2}\left(y_{\beta}^{-}\right)+\zeta_{2}\right\}+\mathcal{O}\left(\epsilon^{0}\right) \text {, } \\
& f_{A B}^{g g}(\beta, \epsilon)=\frac{1}{\epsilon^{3}} \frac{1}{8 \beta}\left\{3 \beta+2 z_{\beta} \ln \left(x_{\beta}\right)\right\}-\frac{1}{\epsilon^{2}} \frac{1}{24 \beta^{2}}\left\{32 \beta^{2}+\beta\left(31+13 \beta^{2}\right) \ln \left(x_{\beta}\right)\right. \\
& \left.+12 z_{\beta} \beta\left[\operatorname{Li}_{2}(\beta)-\mathrm{Li}_{2}(-\beta)\right]+3 z_{\beta}^{2} \ln ^{2}\left(x_{\beta}\right)\right\} \\
& -\frac{1}{\epsilon} \frac{1}{72 \beta^{2}}\left\{104 \beta^{2}+27 z_{\beta}^{2} \zeta_{3}-120 \beta^{2} \ln (2)\right. \\
& +36 z_{\beta}^{2}\left(\operatorname{Li}_{3}\left(x_{\beta}\right)-\mathrm{Li}_{3}\left(y_{\beta}^{-}\right)-\mathrm{Li}_{3}\left(y_{\beta}^{+}\right)\right)+72 \beta z_{\beta}\left(\operatorname{Li}_{3}(\beta)-\mathrm{Li}_{3}(-\beta)\right) \\
& +2 \beta\left(62 \beta^{2}-25\right) \ln \left(x_{\beta}\right)-12 \beta\left(4 \beta^{2}+13\right)\left(\operatorname{Li}_{2}(\beta)-\operatorname{Li}_{2}(-\beta)\right) \\
& +6 \beta\left(\beta^{2}-2\right)\left(\zeta_{2}-2 \operatorname{Li}_{2}\left(y_{\beta}^{-}\right)-\ln ^{2}\left(y_{\beta}^{-}\right)\right) \\
& -18 z_{\beta}^{2} \ln \left(x_{\beta}\right)\left(\mathrm{Li}_{2}(\beta)-\mathrm{Li}_{2}(-\beta)\right) \\
& -3\left(24+2 \beta+9 \beta^{2}-\beta^{3}+12 \beta^{4}\right) \ln ^{2}\left(x_{\beta}\right) \\
& -132 \beta z_{\beta} \ln (2) \ln \left(x_{\beta}\right)-18 \zeta_{2} z_{\beta}^{2}\left(3 \ln \left(x_{\beta}\right)-2 \ln \left(y_{\beta}^{-}\right)\right) \\
& +18 z_{\beta}^{2} \ln (\beta) \ln ^{2}\left(x_{\beta}\right)+6 z_{\beta}\left(3+2 \beta+3 \beta^{2}\right) \ln ^{3}\left(x_{\beta}\right) \\
& \left.+6 z_{\beta}^{2}\left(3 \ln \left(x_{\beta}\right) \ln ^{2}\left(y_{\beta}^{-}\right)-2 \ln ^{3}\left(y_{\beta}^{-}\right)-6 \ln ^{2}\left(x_{\beta}\right) \ln \left(y_{\beta}^{-}\right)\right)\right\}+\mathcal{O}\left(\epsilon^{0}\right) \text {, } \\
& f_{A A}^{q \bar{q}}(\beta, \epsilon)=-\frac{1}{4 \epsilon^{2}}+\frac{1}{\epsilon} \frac{1}{4 \beta}\left\{6 \beta-4 \beta \ln (2)+\ln \left(x_{\beta}\right)\right\}+\mathcal{O}\left(\epsilon^{0}\right), \\
& f_{A B}^{q \bar{q}}(\beta, \epsilon)=\frac{1}{\epsilon^{2}} \frac{1}{12 \beta}\left\{z_{\beta} \ln \left(x_{\beta}\right)-\beta\right\}+\frac{1}{\epsilon} \frac{1}{72 \beta}\left\{34 \beta-\left(37 \beta^{2}+43\right) \ln \left(x_{\beta}\right)\right. \\
& -24 z_{\beta}\left(\operatorname{Li}_{2}\left(y_{\beta}^{-}\right)+\operatorname{Li}_{2}(\beta)-\mathrm{Li}_{2}(-\beta)\right)-24 \beta \ln (2) \\
& \left.+6 z_{\beta}\left(\ln ^{2}\left(x_{\beta}\right)-2 \ln ^{2}\left(y_{\beta}^{-}\right)+4 \ln (2) \ln \left(x_{\beta}\right)+2 \zeta_{2}\right)\right\}+\mathcal{O}\left(\epsilon^{0}\right)
\end{aligned}
$$

where we used the abbreviations

$$
x_{\beta}=\frac{1-\beta}{1+\beta}, \quad y_{\beta}^{ \pm}=\frac{1 \pm \beta}{2}, \quad z_{\beta}=1+\beta^{2} .
$$

Even though expressions for the finite parts of the functions $f_{i j}^{g g, q \bar{q}}(\beta, \epsilon)$ are rather long, they simplify in certain limits. In what follows, we present the expansions in the threshold limit, $\beta \rightarrow 0$, and the high-energy limit, $\beta \rightarrow 1$.

We begin with the threshold limit, where the energies of the emitting quarks are close to their masses, i.e. $E \approx m$, which implies $\beta \ll 1$. We perform a Taylor expansion in small 
$\beta$ and find

$$
\begin{aligned}
f_{A A}^{g g}(\beta \approx 0, \epsilon)= & -\frac{1}{8 \epsilon^{3}}-\frac{1}{4 \epsilon^{2}}+\frac{1-2 \ln (2)}{\epsilon}+2\left(2 \ln (2)-1-\frac{\pi^{2}}{6}\right) \\
& +\beta^{2}\left[-\frac{1}{6 \epsilon^{2}}-\frac{4}{9 \epsilon}+\left(\frac{1}{27}-\frac{8}{3} \ln (2)\right)\right]+\mathcal{O}\left(\beta^{4}\right) \\
f_{A B}^{g g}(\beta \approx 0, \epsilon)= & -\frac{1}{8 \epsilon^{3}}-\frac{1}{4 \epsilon^{2}}+\frac{1-2 \ln (2)}{\epsilon}+2\left(2 \ln (2)-1-\frac{\pi^{2}}{6}\right) \\
& +\beta^{2}\left[-\frac{2}{3 \epsilon^{3}}-\frac{1}{2 \epsilon^{2}}+\frac{1}{\epsilon}\left(1-\frac{44}{9} \ln (2)\right)\right. \\
& \left.+\left(\frac{104}{27} \ln (2)-\frac{1}{3}-\frac{22}{27} \pi^{2}\right)\right]+\mathcal{O}\left(\beta^{4}\right) \\
f_{A A}^{q \bar{q}}(\beta \approx 0, \epsilon)= & -\frac{1}{4 \epsilon^{2}}+\frac{1-\ln (2)}{\epsilon}+\left(4 \ln (2)-\frac{3}{2}-\frac{\pi^{2}}{6}\right) \\
& +\beta^{2}\left[-\frac{1}{6 \epsilon}+\left(\frac{13}{18}-\frac{4}{3} \ln (2)\right)\right]+\mathcal{O}\left(\beta^{4}\right) \\
& +\frac{1}{4 \epsilon^{2}}+\frac{1-\ln (2)}{\epsilon}+\left(4 \ln (2)-\frac{3}{2}-\frac{\pi^{2}}{6}\right) \\
& +\beta^{2}\left[-\frac{2}{9 \epsilon^{2}}+\frac{1}{\epsilon}\left(\frac{25}{54}-\frac{8}{9} \ln (2)\right)+\left(\frac{23}{162}-\frac{4}{27} \pi^{2}+\frac{44}{27} \ln (2)\right)\right] \\
& +\mathcal{O}\left(\beta^{4}\right) .
\end{aligned}
$$

Note that the leading terms in eqs. (4.8)-(4.11) are equal for emitters in a back-to-back kinematics $(A B)$ and self-correlated emissions $(A A)$, i.e.

$$
f_{A A}^{g g, q \bar{q}}(\beta, \epsilon)=f_{A B}^{g g, q \bar{q}}(\beta, \epsilon)+\mathcal{O}\left(\beta^{2}\right) .
$$

This is the case, since in the threshold limit, $\beta=0$, the spatial parts of momenta $p_{A}$ and $p_{B}$ vanish.

In the high-energy limit, the energies of the emitting quarks are much larger than their masses, $E \gg m$, which implies $\beta \approx 1$. Expanding in $(1-\beta)$, we find

$$
\begin{aligned}
f_{A A}^{g g}(\beta \approx 1, \epsilon)= & -\frac{1}{8 \epsilon^{3}}+\frac{1-\ln (2)}{4 \epsilon^{2}}+\frac{1}{2 \epsilon}\left(1-\frac{\pi^{2}}{6}-\frac{5}{2} \ln (2)-\frac{1}{2} \ln ^{2}(2)\right) \\
& +\left(\frac{21}{2} \ln (2)-3-\frac{\pi^{2}}{6} \ln (2)-\frac{\pi^{2}}{24}-\frac{1}{6} \ln ^{3}(2)-\frac{7}{4} \ln ^{2}(2)-\frac{\zeta_{3}}{2}\right) \\
& +\ln (1-\beta)\left[\frac{1}{4 \epsilon^{2}}+\frac{1}{\epsilon}\left(\frac{1}{2} \ln (2)-\frac{3}{4}\right)+\left(\frac{\pi^{2}}{6}-\frac{1}{2}+3 \ln (2)+\frac{1}{2} \ln ^{2}(2)\right)\right] \\
& -\ln ^{2}(1-\beta)\left[\frac{1}{4 \epsilon}+\left(\frac{1}{2} \ln (2)-\frac{3}{4}\right)\right]+\frac{1}{6} \ln ^{3}(1-\beta)+\mathcal{O}(1-\beta), \\
f_{A B}^{g g}(\beta \approx 1, \epsilon)= & \frac{1}{\epsilon^{3}}\left(\frac{3}{8}-\frac{1}{2} \ln (2)\right)+\frac{1}{\epsilon^{2}}\left(\frac{11}{6} \ln (2)-\frac{4}{3}-\frac{\pi^{2}}{4}-\frac{1}{2} \ln ^{2}(2)\right) \\
& +\frac{1}{\epsilon}\left(\frac{13 \pi^{2}}{18}-3 \zeta_{3}-\frac{13}{9}-\frac{1}{3} \ln ^{3}(2)-\frac{11}{6} \ln ^{2}(2)+\frac{97}{36} \ln (2)-\frac{5 \pi^{2}}{12} \ln (2)\right)
\end{aligned}
$$




$$
\begin{aligned}
+ & \left(6 \operatorname{Li}_{4}\left(\frac{1}{2}\right)+\frac{7 \zeta_{3}}{3}+\frac{5 \zeta_{3}}{2} \ln (2)+\frac{1787}{108}+\frac{179 \pi^{2}}{108}-\frac{13 \pi^{4}}{48}+\frac{1}{12} \ln ^{4}(2)\right. \\
& \left.+\frac{11}{9} \ln ^{3}(2)+\frac{881}{36} \ln ^{2}(2)-\frac{2 \pi^{2}}{3} \ln ^{2}(2)-\frac{2059}{54} \ln (2)-\frac{13 \pi^{2}}{18} \ln (2)\right) \\
+ & \ln (1-\beta)\left[\frac{1}{2 \epsilon^{3}}+\frac{1}{\epsilon^{2}}\left(\ln (2)-\frac{11}{6}\right)+\frac{1}{\epsilon}\left(\ln ^{2}(2)+\frac{5 \pi^{2}}{12}-\frac{37}{36}\right)\right. \\
& \left.+\left(\frac{11 \zeta_{3}}{4}+\frac{491}{27}-\frac{10 \pi^{2}}{9}+\frac{2}{3} \ln ^{3}(2)-\frac{163}{6} \ln (2)+\frac{5 \pi^{2}}{6} \ln (2)\right)\right] \\
+ & \ln ^{2}(1-\beta)\left[-\frac{1}{2 \epsilon^{2}}-\frac{1}{\epsilon}\left(\ln (2)-\frac{11}{6}\right)-\left(\ln ^{2}(2)+\frac{5 \pi^{2}}{12}-\frac{37}{36}\right)\right] \\
+ & \ln ^{3}(1-\beta)\left[\frac{1}{3 \epsilon}+\left(\frac{2}{3} \ln (2)-\frac{11}{9}\right)\right]-\frac{1}{6} \ln ^{4}(1-\beta)+\mathcal{O}(1-\beta), \\
f_{A A}^{q \bar{q}}(\beta \approx 1, \epsilon)=- & \frac{1}{4 \epsilon^{2}}+\frac{1}{\epsilon}\left(\frac{3}{2}-\frac{5}{4} \ln (2)\right)+\left(\frac{43}{4} \ln (2)-\frac{7}{4} \ln ^{2}(2)-6-\frac{5 \pi^{2}}{24}\right) \\
+ & \ln (1-\beta)\left[\frac{1}{4 \epsilon}+\left(3 \ln (2)-\frac{11}{4}\right)\right]-\frac{1}{4} \ln ^{2}(1-\beta)+\mathcal{O}(1-\beta) \\
f_{A B}^{q \bar{q}}(\beta \approx 1, \epsilon)=- & \frac{1}{\epsilon^{2}}\left(\frac{1}{12}+\frac{1}{6} \ln (2)\right)+\frac{1}{\epsilon}\left(\frac{17}{36}-\frac{\pi^{2}}{9}-\frac{5}{6} \ln ^{2}(2)+\frac{7}{9} \ln (2)\right) \\
+ & \left(\frac{77 \pi^{2}}{108}-\frac{13 \zeta_{3}}{6}-\frac{161}{54}-\frac{1}{9} \ln ^{3}(2)+\frac{44}{9} \ln ^{2}(2)+\frac{31}{27} \ln (2)-\frac{5 \pi^{2}}{9} \ln (2)\right) \\
+ & \ln (1-\beta)\left[\frac{1}{6 \epsilon^{2}}+\frac{1}{\epsilon}\left(\ln (2)-\frac{10}{9}\right)+\left(\frac{139}{54}+\frac{2 \pi^{2}}{9}+\ln { }^{2}(2)-\frac{17}{3} \ln (2)\right)\right] \\
- & \left.\ln { }^{2}(1-\beta)\left[\frac{1}{6 \epsilon}+\left(\ln (2)-\frac{10}{9}\right)\right]+\frac{1}{9} \ln ^{3}(1-\beta)+\mathcal{O}(1-\beta) .15\right)
\end{aligned}
$$

Note that these expressions contain logarithms of the form $\ln ^{n}(1-\beta)$, which are divergent in the $\beta \rightarrow 1$ limit. These logarithms are related to quasi-collinear divergences that appear once the mass of the emitter, which screens the actual collinear divergences, becomes small compared to the overall energy. In the massless calculation [50], all $\ln (1-\beta)$ terms manifest themselves as additional poles in $1 / \epsilon$.

\section{Conclusions}

In this paper, we presented analytic results for the integrated double-soft subtraction terms that are needed in the context of the nested soft-collinear subtraction scheme [49] to describe production of two equal-mass back-to-back partons. Integration over the phase space of unresolved radiation, subject to constraints dictated by the subtraction scheme, was performed using reverse unitarity [62] that allowed us to map phase-space integrals onto conventional loop integrals with cut propagators, and apply standard IBP techniques for the reduction of the integrands to master integrals. These master integrals were computed by solving a corresponding system of differential equations in an $\epsilon$-homogeneous form.

The resulting subtraction terms provide an essential ingredient for NNLO calculations featuring massive partons. We note that it is possible to obtain these integrated subtraction 
terms numerically, as it was done, for example, in refs. [30, 55]. Nevertheless, it is usually beneficial to have analytic results available. The results presented in this article provide all integrated double-soft subtraction terms required for a description of colour-singlet decays into massive fermions. For the case of heavy-quark pair production, it is also necessary to consider integrated subtraction terms with one massless and one massive parton which are not necessarily in a back-to-back kinematics. We leave this problem for future investigations.

\section{Acknowledgments}

We wish to thank Arnd Behring for providing numerous numerical checks and Florian Herren for fruitful discussions. We would like to thank Kirill Melnikov and Fabrizio Caola for valuable feedback on the manuscript. This research is partially supported by the Deutsche Forschungsgemeinschaft (DFG, German Research Foundation) under grant 396021762 TRR 257.

Open Access. This article is distributed under the terms of the Creative Commons Attribution License (CC-BY 4.0), which permits any use, distribution and reproduction in any medium, provided the original author(s) and source are credited.

\section{References}

[1] ATLAS collaboration, Observation of a new particle in the search for the Standard Model Higgs boson with the ATLAS detector at the LHC, Phys. Lett. B 716 (2012) 1 [arXiv: 1207.7214] [INSPIRE].

[2] CMS collaboration, Observation of a new boson at a mass of $125 \mathrm{GeV}$ with the CMS experiment at the LHC, Phys. Lett. B $\mathbf{7 1 6}$ (2012) 30 [arXiv:1207.7235] [INSPIRE].

[3] K. Fabricius, I. Schmitt, G. Kramer and G. Schierholz, Higher order perturbative QCD calculation of jet cross-sections in $e^{+} e^{-}$annihilation, Z. Phys. C 11 (1981) 315 [INSPIRE].

[4] R. Ellis, D.A. Ross and A.E. Terrano, The perturbative calculation of jet structure in $e^{+} e^{-}$ annihilation, Nucl. Phys. B 178 (1981) 421 [INSPIRE].

[5] S. Frixione, Z. Kunszt and A. Signer, Three jet cross-sections to next-to-leading order, Nucl. Phys. B 467 (1996) 399 [hep-ph/9512328] [INSPIRE].

[6] S. Frixione, A general approach to jet cross-sections in QCD, Nucl. Phys. B 507 (1997) 295 [hep-ph/9706545] [INSPIRE].

[7] S. Catani and M.H. Seymour, A general algorithm for calculating jet cross-sections in NLO QCD, Nucl. Phys. B 485 (1997) 291 [Erratum ibid. 510 (1998) 503] [hep-ph/9605323] [INSPIRE].

[8] S. Catani, S. Dittmaier, M.H. Seymour and Z. Trócsányi, The dipole formalism for next-to-leading order QCD calculations with massive partons, Nucl. Phys. B 627 (2002) 189 [hep-ph/0201036] [INSPIRE].

[9] S. Catani and M. Grazzini, An NNLO subtraction formalism in hadron collisions and its application to Higgs boson production at the LHC, Phys. Rev. Lett. 98 (2007) 222002 [hep-ph/0703012] [INSPIRE]. 
[10] R. Bonciani, S. Catani, M. Grazzini, H. Sargsyan and A. Torre, The $q_{T}$ subtraction method for top quark production at hadron colliders, Eur. Phys. J. C $\mathbf{7 5}$ (2015) 581 [arXiv: 1508.03585] [INSPIRE].

[11] M. Grazzini, S. Kallweit and M. Wiesemann, Fully differential NNLO computations with MATRIX, Eur. Phys. J. C 78 (2018) 537 [arXiv:1711.06631] [InSPIRE].

[12] S. Catani, S. Devoto, M. Grazzini, S. Kallweit and J. Mazzitelli, Top-quark pair production at the LHC: fully differential QCD predictions at NNLO, JHEP 07 (2019) 100 [arXiv: 1906.06535] [INSPIRE].

[13] R. Boughezal, C. Focke, X. Liu and F. Petriello, $W$-boson production in association with a jet at next-to-next-to-leading order in perturbative QCD, Phys. Rev. Lett. 115 (2015) 062002 [arXiv: 1504.02131] [INSPIRE].

[14] R. Boughezal, C. Focke, W. Giele, X. Liu and F. Petriello, Higgs boson production in association with a jet at NNLO using jettiness subtraction, Phys. Lett. B 748 (2015) 5 [arXiv: 1505. 03893] [INSPIRE].

[15] J. Gaunt, M. Stahlhofen, F.J. Tackmann and J.R. Walsh, $N$-jettiness subtractions for NNLO QCD calculations, JHEP 09 (2015) 058 [arXiv: 1505. 04794] [INSPIRE].

[16] R. Boughezal et al., Color singlet production at NNLO in MCFM, Eur. Phys. J. C 77 (2017) 7 [arXiv: 1605. 08011] [INSPIRE].

[17] A. Gehrmann-De Ridder, T. Gehrmann and E.W. Glover, Antenna subtraction at NNLO, JHEP 09 (2005) 056 [hep-ph/0505111] [INSPIRE].

[18] A. Gehrmann-De Ridder, T. Gehrmann and E.W. Glover, Gluon-gluon antenna functions from Higgs boson decay, Phys. Lett. B 612 (2005) 49 [hep-ph/0502110] [InSPIRE].

[19] A. Gehrmann-De Ridder, T. Gehrmann and E.W. Glover, Quark-gluon antenna functions from neutralino decay, Phys. Lett. B 612 (2005) 36 [hep-ph/0501291] [INSPIRE].

[20] A. Daleo, T. Gehrmann and D. Maître, Antenna subtraction with hadronic initial states, JHEP 04 (2007) 016 [hep-ph/0612257] [INSPIRE].

[21] A. Daleo, A. Gehrmann-De Ridder, T. Gehrmann and G. Luisoni, Antenna subtraction at NNLO with hadronic initial states: initial-final configurations, JHEP 01 (2010) 118 [arXiv:0912.0374] [INSPIRE].

[22] T. Gehrmann and P.F. Monni, Antenna subtraction at NNLO with hadronic initial states: real-virtual initial-initial configurations, JHEP 12 (2011) 049 [arXiv:1107.4037] [INSPIRE].

[23] R. Boughezal, A. Gehrmann-De Ridder and M. Ritzmann, Antenna subtraction at NNLO with hadronic initial states: double real radiation for initial-initial configurations with two quark flavours, JHEP 02 (2011) 098 [arXiv: 1011.6631] [inSPIRE].

[24] A. Gehrmann-De Ridder, T. Gehrmann and M. Ritzmann, Antenna subtraction at NNLO with hadronic initial states: double real initial-initial configurations, JHEP 10 (2012) 047 [arXiv: 1207.5779] [INSPIRE].

[25] J. Currie, E.W.N. Glover and S. Wells, Infrared structure at NNLO using antenna subtraction, JHEP 04 (2013) 066 [arXiv:1301.4693] [INSPIRE].

[26] J. Currie, A. Gehrmann-De Ridder, T. Gehrmann, E.W.N. Glover, A. Huss and J. Pires, Precise predictions for dijet production at the LHC, Phys. Rev. Lett. 119 (2017) 152001 [arXiv: 1705.10271] [INSPIRE]. 
[27] J. Currie, A. Gehrmann-De Ridder, T. Gehrmann, E.W.N. Glover, A. Huss and J. Pires, Infrared sensitivity of single jet inclusive production at hadron colliders, JHEP 10 (2018) 155 [arXiv: 1807.03692] [INSPIRE].

[28] F. Herzog, Geometric IR subtraction for final state real radiation, JHEP 08 (2018) 006 [arXiv: 1804.07949] [INSPIRE].

[29] M. Czakon, A novel subtraction scheme for double-real radiation at NNLO, Phys. Lett. B 693 (2010) 259 [arXiv: 1005. 0274] [INSPIRE].

[30] M. Czakon, Double-real radiation in hadronic top quark pair production as a proof of a certain concept, Nucl. Phys. B 849 (2011) 250 [arXiv:1101.0642] [InSPIRE].

[31] M. Czakon and D. Heymes, Four-dimensional formulation of the sector-improved residue subtraction scheme, Nucl. Phys. B 890 (2014) 152 [arXiv:1408.2500] [inSPIRE].

[32] M. Czakon, A. van Hameren, A. Mitov and R. Poncelet, Single-jet inclusive rates with exact color at $O\left(\alpha_{s}^{4}\right)$, JHEP 10 (2019) 262 [arXiv:1907.12911] [inSPIRE].

[33] H.A. Chawdhry, M.L. Czakon, A. Mitov and R. Poncelet, NNLO QCD corrections to three-photon production at the LHC, JHEP 02 (2020) 057 [arXiv: 1911. 00479] [INSPIRE].

[34] L. Magnea, E. Maina, G. Pelliccioli, C. Signorile-Signorile, P. Torrielli and S. Uccirati, Local analytic sector subtraction at NNLO, JHEP 12 (2018) 107 [Erratum ibid. 06 (2019) 013] [arXiv: 1806.09570] [INSPIRE].

[35] L. Magnea, E. Maina, G. Pelliccioli, C. Signorile-Signorile, P. Torrielli and S. Uccirati, Factorisation and subtraction beyond NLO, JHEP 12 (2018) 062 [arXiv:1809.05444] [INSPIRE].

[36] G. Somogyi, Z. Trócsányi and V. Del Duca, Matching of singly- and doubly-unresolved limits of tree-level QCD squared matrix elements, JHEP 06 (2005) 024 [hep-ph/0502226] [INSPIRE].

[37] G. Somogyi and Z. Trócsányi, A new subtraction scheme for computing QCD jet cross sections at next-to-leading order accuracy, hep-ph/0609041 [INSPIRE].

[38] G. Somogyi, Z. Trócsányi and V. Del Duca, A subtraction scheme for computing QCD jet cross sections at NNLO: regularization of doubly-real emissions, JHEP 01 (2007) 070 [hep-ph/0609042] [INSPIRE].

[39] G. Somogyi and Z. Trócsányi, A subtraction scheme for computing QCD jet cross sections at NNLO: regularization of real-virtual emission, JHEP 01 (2007) 052 [hep-ph/0609043] [INSPIRE].

[40] G. Somogyi and Z. Trócsányi, A subtraction scheme for computing QCD jet cross sections at NNLO: integrating the subtraction terms. I, JHEP 08 (2008) 042 [arXiv:0807.0509] [INSPIRE].

[41] U. Aglietti, V. Del Duca, C. Duhr, G. Somogyi and Z. Trócsányi, Analytic integration of real-virtual counterterms in NNLO jet cross sections. I, JHEP 09 (2008) 107 [arXiv: 0807.0514] [INSPIRE].

[42] G. Somogyi, Subtraction with hadronic initial states at NLO: an NNLO-compatible scheme, JHEP 05 (2009) 016 [arXiv:0903.1218] [INSPIRE].

[43] P. Bolzoni, S.-O. Moch, G. Somogyi and Z. Trócsányi, Analytic integration of real-virtual counterterms in NNLO jet cross sections. II, JHEP 08 (2009) 079 [arXiv:0905.4390] [INSPIRE]. 
[44] P. Bolzoni, G. Somogyi and Z. Trócsányi, A subtraction scheme for computing QCD jet cross sections at NNLO: integrating the iterated singly-unresolved subtraction terms, JHEP 01 (2011) 059 [arXiv: 1011.1909] [INSPIRE].

[45] V. Del Duca, G. Somogyi and Z. Trócsányi, Integration of collinear-type doubly unresolved counterterms in NNLO jet cross sections, JHEP 06 (2013) 079 [arXiv:1301.3504] [INSPIRE].

[46] G. Somogyi, A subtraction scheme for computing QCD jet cross sections at NNLO: integrating the doubly unresolved subtraction terms, JHEP 04 (2013) 010 [arXiv: 1301.3919] [INSPIRE].

[47] V. Del Duca et al., Jet production in the CoLoRFulNNLO method: event shapes in electron-positron collisions, Phys. Rev. D 94 (2016) 074019 [arXiv: 1606. 03453] [INSPIRE].

[48] M. Cacciari, F.A. Dreyer, A. Karlberg, G.P. Salam and G. Zanderighi, Fully differential vector-boson-fusion Higgs production at next-to-next-to-leading order, Phys. Rev. Lett. 115 (2015) 082002 [Erratum ibid. 120 (2018) 139901] [arXiv: 1506.02660] [INSPIRE].

[49] F. Caola, K. Melnikov and R. Röntsch, Nested soft-collinear subtractions in NNLO QCD computations, Eur. Phys. J. C 77 (2017) 248 [arXiv:1702.01352] [INSPIRE].

[50] F. Caola, M. Delto, H. Frellesvig and K. Melnikov, The double-soft integral for an arbitrary angle between hard radiators, Eur. Phys. J. C 78 (2018) 687 [arXiv:1807.05835] [INSPIRE].

[51] M. Delto and K. Melnikov, Integrated triple-collinear counter-terms for the nested soft-collinear subtraction scheme, JHEP 05 (2019) 148 [arXiv: 1901.05213] [INSPIRE].

[52] F. Caola, K. Melnikov and R. Röntsch, Analytic results for color-singlet production at NNLO QCD with the nested soft-collinear subtraction scheme, Eur. Phys. J. C 79 (2019) 386 [arXiv: 1902.02081] [INSPIRE].

[53] F. Caola, K. Melnikov and R. Röntsch, Analytic results for decays of color singlets to gg and $q \bar{q}$ final states at NNLO QCD with the nested soft-collinear subtraction scheme, Eur. Phys. J. C 79 (2019) 1013 [arXiv: 1907.05398] [InSPIRE].

[54] K. Asteriadis, F. Caola, K. Melnikov and R. Röntsch, Analytic results for deep-inelastic scattering at NNLO QCD with the nested soft-collinear subtraction scheme, Eur. Phys. J. C 80 (2020) 8 [arXiv: 1910.13761] [INSPIRE].

[55] A. Behring and W. Bizoń, Higgs decay into massive b-quarks at NNLO QCD in the nested soft-collinear subtraction scheme, JHEP 01 (2020) 189 [arXiv:1911.11524] [INSPIRE].

[56] S. Catani and M. Grazzini, Infrared factorization of tree level QCD amplitudes at the next-to-next-to-leading order and beyond, Nucl. Phys. B 570 (2000) 287 [hep-ph/9908523] [INSPIRE].

[57] G. Wang, X. Xu, L.L. Yang and H.X. Zhu, The next-to-next-to-leading order soft function for top quark pair production, JHEP 06 (2018) 013 [arXiv: 1804.05218] [INSPIRE].

[58] M. Abramowitz and I.A. Stegun, Handbook of mathematical functions with formulas, graphs and mathematical tables, Dover, U.S.A. (1964).

[59] T. Huber and D. Maître, HypExp 2, expanding hypergeometric functions about half-integer parameters, Comput. Phys. Commun. 178 (2008) 755 [arXiv:0708.2443] [INSPIRE].

[60] S. Alioli, P. Nason, C. Oleari and E. Re, A general framework for implementing NLO calculations in shower Monte Carlo programs: the POWHEG BOX, JHEP 06 (2010) 043 [arXiv: 1002.2581] [INSPIRE]. 
[61] G. Somogyi, Angular integrals in d dimensions, J. Math. Phys. 52 (2011) 083501 [arXiv: 1101.3557] [INSPIRE].

[62] C. Anastasiou and K. Melnikov, Higgs boson production at hadron colliders in NNLO QCD, Nucl. Phys. B 646 (2002) 220 [hep-ph/0207004] [INSPIRE].

[63] R.E. Cutkosky, Singularities and discontinuities of Feynman amplitudes, J. Math. Phys. 1 (1960) 429 [INSPIRE].

[64] K.G. Chetyrkin and F.V. Tkachov, Integration by parts: the algorithm to calculate $\beta$-functions in 4 loops, Nucl. Phys. B 192 (1981) 159 [INSPIRE].

[65] A. von Manteuffel and C. Studerus, Reduze 2 - distributed Feynman integral reduction, arXiv: 1201.4330 [INSPIRE].

[66] A.V. Kotikov, Differential equations method: new technique for massive Feynman diagrams calculation, Phys. Lett. B 254 (1991) 158 [InSPIRE].

[67] E. Remiddi, Differential equations for Feynman graph amplitudes, Nuovo Cim. A 110 (1997) 1435 [hep-th/9711188] [INSPIRE].

[68] T. Gehrmann and E. Remiddi, Differential equations for two loop four point functions, Nucl. Phys. B 580 (2000) 485 [hep-ph/9912329] [INSPIRE].

[69] J.M. Henn, Multiloop integrals in dimensional regularization made simple, Phys. Rev. Lett. 110 (2013) 251601 [arXiv: 1304.1806] [INSPIRE].

[70] C. Meyer, Transforming differential equations of multi-loop Feynman integrals into canonical form, JHEP 04 (2017) 006 [arXiv: 1611.01087] [INSPIRE].

[71] C. Meyer, Algorithmic transformation of multi-loop master integrals to a canonical basis with CANONICA, Comput. Phys. Commun. 222 (2018) 295 [arXiv:1705. 06252] [INSPIRE].

[72] R.N. Lee, Reducing differential equations for multiloop master integrals, JHEP 04 (2015) 108 [arXiv: 1411.0911] [inSPIRE].

[73] A.B. Goncharov, Polylogarithms in arithmetic and geometry, in Proceedings of the International Congress of Mathematicians, Birkhäuser, Basel, Switzerland (1995), pg. 374.

[74] C. Duhr and F. Dulat, PolyLogTools — polylogs for the masses, JHEP 08 (2019) 135 [arXiv: 1904.07279] [INSPIRE].

[75] H. Frellesvig, Generalized polylogarithms in Maple, arXiv:1806.02883 [INSPIRE].

[76] J. Vollinga and S. Weinzierl, Numerical evaluation of multiple polylogarithms, Comput. Phys. Commun. 167 (2005) 177 [hep-ph/0410259] [INSPIRE].

[77] L. Naterop, A. Signer and Y. Ulrich, handyG - rapid numerical evaluation of generalised polylogarithms in Fortran, Comput. Phys. Commun. 253 (2020) 107165 [arXiv: 1909.01656] [INSPIRE].

[78] E. Remiddi and J.A.M. Vermaseren, Harmonic polylogarithms, Int. J. Mod. Phys. A 15 (2000) 725 [hep-ph/9905237] [INSPIRE].

[79] C. Duhr, H. Gangl and J.R. Rhodes, From polygons and symbols to polylogarithmic functions, JHEP 10 (2012) 075 [arXiv:1110.0458] [INSPIRE]. 